\title{
Hexagonal patterns in finite domains
}

\author{
P.C. Matthews \\ Department of Theoretical Mechanics, University of Nottingham, Nottingham NG7 \\ 2RD, United Kingdom
}

\begin{abstract}
In many mathematical models for pattern formation, a regular hexagonal pattern is stable in an infinite region. However, laboratory and numerical experiments are carried out in finite domains, and this imposes certain constraints on the possible patterns. In finite rectangular domains, it is shown that a regular hexagonal pattern cannot occur if the aspect ratio is rational.

In practice, it is found experimentally that in a rectangular region, patterns of irregular hexagons are often observed. This work analyses the geometry and dynamics of irregular hexagonal patterns. These patterns occur in two different symmetry types, either with a reflection symmetry, involving two wavenumbers, or without symmetry, involving three different wavenumbers.

The relevant amplitude equations are studied to investigate the detailed bifurcation structure in each case. It is shown that hexagonal patterns can bifurcate subcritically either from the trivial solution or from a pattern of rolls.

Numerical simulations of a model partial differential equation are also presented to illustrate the behaviour.
\end{abstract}

Key words: Patterns, hexagons, bifurcations, dynamics.

\section{Introduction}

Pattern formation is a topic of intensive current research; a recent volume of this journal has been dedicated to the subject [14]. There are many experimental examples of pattern formation, including Rayleigh-Benard convection [6], directional solidification [8] and the Faraday experiment [11]. It is now appreciated that the amplitude equations describing the evolution and dynamics of patterns are generic, so that many different physical systems are governed by the same equations. These amplitude equations and their possible solutions are determined more by the symmetries of the problem than the details of the physics [3]. 
Theoretical work on pattern formation has generally been concerned with regions of infinite horizontal extent, with attention focussed on modes with a single wavenumber. Analysis of the competition between rolls and hexagons shows that in systems without an up-down symmetry, hexagons are generally preferred. For hexagons, a sign change in the pattern gives a genuinely different solution, while for rolls a sign change is equivalent to a spatial translation of half a wavelength. This means that hexagons can occur at a transcritical bifurcation but rolls can only appear at a pitchfork bifurcation. In systems with an up-down symmetry, only pitchfork bifurcations occur, and either rolls, squares, or hexagons may be stable, depending on the coefficients in the amplitude equations.

Recently, more complicated patterns involving four sets of rolls with the same wavenumber have been considered [5]. These share the symmetry properties of rolls and squares, so a sign change is equivalent to a spatial shift.

Analysis of patterns involving a single wavenumber is not always successful in predicting patterns. Resonant mode interactions can be important near certain points in parameter space. These resonances generate additional quadratic terms in the amplitude equations and can lead to more exotic patterns with complicated dynamics [15].

In most examples of experimental interest, there is some up-down asymmetry which leads to a preference for hexagons at the onset of instability. However, the finite geometry of the experimental system or numerical simulation does not in general allow a regular hexagonal pattern. What is observed in practice is a solution which is close to regular hexagons, but in fact involves slightly different wavenumbers. An example is the numerical experiments on compressible magnetoconvection by Weiss et al. (1996) [18], who found patterns of non-regular hexagons in square domains. Laboratory experiments on surface-tension-driven convection in square container also yield a variety of puzzling patterns [9].

Such irregular or non-equilateral hexagons can also occur in a region of infinite horizontal extent with anisotropy, since breaking rotational invariance also breaks the symmetry of the hexagons. An example of this is convection influenced by a weak shear flow [7,1]. Another application is in the numerical modelling of hexagonal patterns [16]; if the numerical method does not preserve the symmetry of the hexagons then a qualitatively incorrect bifurcation diagram can be obtained.

Theoretical work on non-equilateral hexagons has been carried out by Malomed, Nepomnyashchy and Nuz [12], who studied the governing amplitude equations in two cases. If the pattern involves two equal wavenumbers, a hexagonal pattern can be stable in a region of parameter space near the onset of 
instability, and can branch either directly from the trivial solution or from a roll pattern. In the case of three different wavenumbers, hexagons can only be stable at finite amplitude. The same amplitude equations have also been studied in the context of convection in the presence of a mean flow $[7,1]$, and for an anisotropic solidification problem [8]. However, none of these works has provided complete bifurcation diagrams showing how the well-known picture for competition between rolls and regular hexagons is modified by the anisotropy.

This paper describes the geometry of irregular hexagonal patterns in section 2 , considering the constraints imposed by a finite domain and relating the wavenumbers involved in the pattern to the number of hexagons seen. Section 3 considers the nonlinear dynamics of these patterns, concentrating on those aspects of the problem not covered by previous work. To illustrate the possible types of behaviour and to provide a check on the analytical work, numerical simulations of the modified Swift-Hohenberg model [17] are described in section 4.

\section{Geometry of hexagonal patterns}

This section describes the geometry of hexagonal patterns that can be obtained in a square box with sides of length $L$ with either periodic or Neumann boundary conditions.

For periodic boundary conditions, any pattern $w(x, y)$ can be written as a Fourier series,

$$
w=\sum_{m=1}^{\infty} \sum_{n=1}^{\infty} A_{m n} \exp 2 \pi i(m x+n y) / L
$$

and for Neumann boundary conditions $\left(\frac{\partial w}{\partial x}=0\right.$ at $x=0, L ; \frac{\partial w}{\partial y}=0$ at $y=0$, $L)$

$$
w=\sum_{m=1}^{\infty} \sum_{n=1}^{\infty} A_{m n}(\cos \pi(m x+n y) / L+\cos \pi(m x-n y) / L) .
$$

Note that the possible patterns for Neumann boundary conditions are a subset of those for periodic boundary conditions in a box twice as large. This fact is often referred to as a 'hidden symmetry' [2,4]. Henceforth periodic boundary conditions will be assumed, since this includes the other case. The integers $m$ and $n$ in (1) will be referred to as 'wave integers'. The corresponding wavenumber is

$$
k=2 \pi \sqrt{m^{2}+n^{2}} / L
$$


A hexagonal pattern is obtained by taking three modes whose wave integers sum to zero, i.e.

$$
m_{1}+m_{2}+m_{3}=0, \quad n_{1}+n_{2}+n_{3}=0 .
$$

This is necessary if 'strong' resonance (i.e. a quadratic term) is to occur in the amplitude equations.

A pattern of regular hexagons with three equal wavenumbers cannot occur in a square box. This result is not immediately obvious (in general the hexagons could be aligned at any angle to the box) but can be demonstrated as follows. If the three wavenumbers are equal then

$$
m_{1}^{2}+n_{1}^{2}=m_{2}^{2}+n_{2}^{2}=m_{3}^{2}+n_{3}^{2},
$$

which can be rewritten using (4) as

$$
m_{1}^{2}+n_{1}^{2}=m_{2}^{2}+n_{2}^{2}=\left(m_{1}+m_{2}\right)^{2}+\left(n_{1}+n_{2}\right)^{2} .
$$

Expanding the brackets, these equations simplify to

$$
m_{1}^{2}+n_{1}^{2}=m_{2}^{2}+n_{2}^{2}=-2\left(m_{1} m_{2}+n_{1} n_{2}\right)
$$

showing that the sums of the squares of the wave integers are even. However it can be stipulated that not all the wave integers are even, since any common factors can be removed. There are two possibilities, each of which lead to a contradiction. If $m_{1}, n_{1}$ are odd and $m_{2}, n_{2}$ are even, then $m_{1}^{2}+n_{1}^{2}=2 \bmod 4$ but $m_{2}^{2}+n_{2}^{2}=0 \bmod 4$. Alternatively if $m_{1}, n_{1}, m_{2}, n_{2}$ are all odd, then $m_{1}^{2}+n_{1}^{2}=2 \bmod 4$ but $2\left(m_{1} m_{2}+n_{1} n_{2}\right)=0 \bmod 4$.

This result, that regular hexagons cannot occur in a square box, generalizes to the case of a rectangular box provided that the aspect ratio of the box is rational. In this case, if the box is of size $L_{1}$ in the $x$-direction and $L_{2}$ in the $y$-direction and $L_{1} / L_{2}=p / q$ where $p$ and $q$ are integers, the constraint of equal wavenumbers requires

$$
m_{1}^{2} / L_{1}^{2}+n_{1}^{2} / L_{2}^{2}=m_{2}^{2} / L_{1}^{2}+n_{2}^{2} / L_{2}^{2}=m_{3}^{2} / L_{1}^{2}+n_{3}^{2} / L_{2}^{2} .
$$

Multiplying through by $L_{1} L_{2} p q$, this becomes

$$
\left(m_{1} q\right)^{2}+\left(n_{1} p\right)^{2}=\left(m_{2} q\right)^{2}+\left(n_{2} p\right)^{2}=\left(m_{3} q\right)^{2}+\left(n_{3} p\right)^{2}
$$

which is equivalent to (5) with $m_{i}$ replaced by $m_{i} q$ and $n_{i}$ replaced by $m_{i} p$, so that the problem has been reduced to the previous case. 
(a)

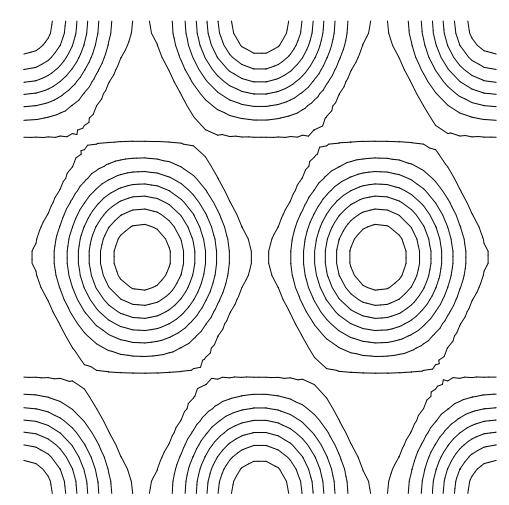

(b)

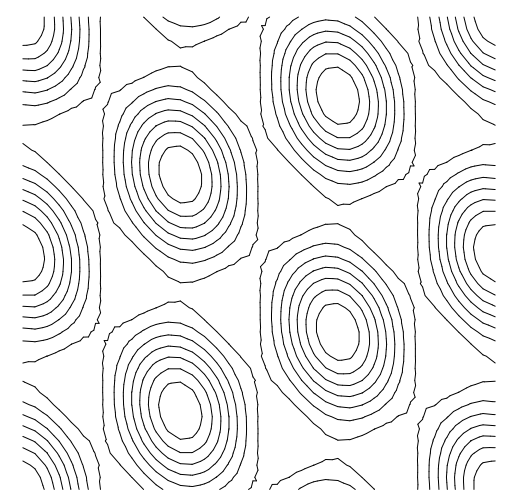

Fig. 1. Hexagonal patterns with wave integers (a) $(2,1),(-2,1),(0,-2) ;(b)(3,0)$, $(-2,-2),(-1,2)$.

Regular hexagons can however be obtained in a rectangular box if the ratio of the length of one side to the other is a multiple of $\sqrt{3}$; this choice has been used in numerical simulations to investigate the relative stability of rolls and hexagons [13].

Two examples of irregular hexagonal patterns are illustrated in Fig. 1. In each case the amplitudes of the three modes have been chosen to be real and equal, so that the function plotted is

$$
w=\sum_{i=1}^{3} \cos \left(2 \pi\left(m_{i} x+n_{i} y\right) / L\right) .
$$

Fig. 1 (a) shows the case where the wave integer pairs are $(2,1),(-2,1)$ and $(0,-2)$, in which four hexagons occur in the box, while the six-hexagon case with wave integers $(3,0),(-2,-2),(-1,2)$ is shown in Fig. 1 (b). The former can occur with periodic or Neumann boundary conditions, but the latter can only occur with periodic boundary conditions. The six-hexagon pattern shown in Fig. 1 (b) has been found in numerical simulations of magnetoconvection in a compressible fluid by Weiss et al. (1996) [18].

It is apparent from these examples that there are two different possible symmetries for these hexagonal patterns. The four-hexagon example, with two equal wavenumbers, has a reflection symmetry $(x \rightarrow-x)$. The six-hexagon case, with three different wavenumbers, does not have a reflection symmetry. It is well known that symmetry has a crucial effect on the nonlinear dynamics of a system (e.g. Crawford and Knobloch 1991 [3]), and it will be shown in section 3 that the bifurcation structure associated with the two patterns is different. 
There is a simple relationship between the number of hexagons $N$ in the periodic box and the wave integers. This is

$$
N=\left|m_{1} n_{2}-m_{2} n_{1}\right|
$$

From (4) it follows that any pair of the wave integers can be used in this formula. This result is demonstrated as follows: the planform function (10) is maximized when $x$ and $y$ obey

$$
m_{1} x+n_{1} y=p L, \quad m_{2} x+n_{2} y=q L,
$$

where $p$ and $q$ are integers. From (4) it follows that $m_{3} x+n_{3} y$ is then also an integer multiple of $L$, so that $w$ takes its maximum value of 3, corresponding to the centre of a hexagon. These equations give the lattice of points at which centres of hexagons occur. Three points on this lattice are

(a) $p=0, q=0, x=0, y=0$;

(b) $p=1, q=0, x=n_{2} L /\left(m_{1} n_{2}-m_{2} n_{1}\right), y=-m_{2} L /\left(m_{1} n_{2}-m_{2} n_{1}\right)$;

(c) $p=0, q=1, x=-n_{1} L /\left(m_{1} n_{2}-m_{2} n_{1}\right), y=m_{1} L /\left(m_{1} n_{2}-m_{2} n_{1}\right)$.

These three points form a triangle that connects the centres of three hexagons.

The area of this triangle is

$$
\left|\left(n_{2},-m_{2}\right) \times\left(-n_{1}, m_{1}\right)\right| L^{2} / 2\left(m_{1} n_{2}-m_{2} n_{1}\right)^{2}=L^{2} / 2\left|m_{1} n_{2}-m_{2} n_{1}\right|,(13
$$

using the formula for the area of a triangle in terms of the cross product of two vectors. This area is half the area associated with each hexagon, since each triangle connects three hexagons but each hexagon is connected to six triangles. Dividing the total area of the square $L^{2}$ by the area of each hexagon gives the formula (11) for the total number of hexagons in the box.

To describe these hexagonal patterns it useful to have a parameter measuring how close the pattern is to regular hexagons. One such parameter is $H=$ $\left(k_{\max }^{2}-k_{\min }^{2}\right) / k_{\max }^{2}$ where $k_{\max }$ and $k_{\min }$ are the maximum and minimum of the three wavenumbers; if $H$ is small, the pattern is close to regular hexagons. Table 1 lists all hexagonal patterns in which $H<0.45$ and $N<25$, giving the wave integers, the parameter $H$, the number of hexagons $N$ and the symmetry for each.

\section{Nonlinear dynamics of hexagonal patterns}

This section describes the behaviour of the nonlinear amplitude equations governing the dynamics of irregular hexagonal patterns. 


\begin{tabular}{ccccccccc}
$m_{1}$ & $n_{1}$ & $m_{2}$ & $n_{2}$ & $m_{3}$ & $n_{3}$ & $H$ & $N$ & Symmetry \\
\hline 2 & 1 & -2 & 1 & 0 & -2 & 0.20000 & 4 & $\mathrm{Y}$ \\
3 & 0 & -2 & 2 & -1 & -2 & 0.44444 & 6 & $\mathrm{~N}$ \\
3 & 1 & -1 & -3 & -2 & 2 & 0.20000 & 8 & $\mathrm{Y}$ \\
3 & 2 & -3 & 1 & 0 & -3 & 0.30769 & 9 & $\mathrm{~N}$ \\
4 & 1 & -3 & 2 & -1 & -3 & 0.41176 & 11 & $\mathrm{~N}$ \\
3 & 3 & -4 & 0 & 1 & -3 & 0.44444 & 12 & $\mathrm{~N}$ \\
4 & 0 & -2 & -3 & -2 & 3 & 0.18750 & 12 & $\mathrm{Y}$ \\
4 & 2 & -1 & -4 & -3 & 2 & 0.35000 & 14 & $\mathrm{~N}$ \\
3 & 3 & -4 & 1 & 1 & -4 & 0.05556 & 15 & $\mathrm{Y}$ \\
4 & 2 & -4 & 2 & 0 & -4 & 0.20000 & 16 & $\mathrm{Y}$ \\
4 & 3 & -4 & 1 & 0 & -4 & 0.36000 & 16 & $\mathrm{~N}$ \\
5 & 1 & -2 & -4 & -3 & 3 & 0.30769 & 18 & $\mathrm{~N}$ \\
5 & 2 & -4 & 2 & -1 & -4 & 0.41379 & 18 & $\mathrm{~N}$ \\
5 & 1 & -4 & 3 & -1 & -4 & 0.34615 & 19 & $\mathrm{~N}$ \\
5 & 0 & -3 & -4 & -2 & 4 & 0.20000 & 20 & $\mathrm{Y}$ \\
5 & 2 & -5 & 2 & 0 & -4 & 0.44828 & 20 & $\mathrm{Y}$ \\
5 & 2 & -2 & -5 & -3 & 3 & 0.37931 & 21 & $\mathrm{Y}$ \\
5 & 3 & -1 & -5 & -4 & 2 & 0.41176 & 22 & $\mathrm{~N}$ \\
5 & 2 & -1 & -5 & -4 & 3 & 0.13793 & 23 & $\mathrm{~N}$ \\
4 & 4 & -5 & 1 & 1 & -5 & 0.18750 & 24 & $\mathrm{Y}$ \\
6 & 0 & -3 & -4 & -3 & 4 & 0.30556 & 24 & $\mathrm{Y}$ \\
6 & 0 & -4 & -4 & -2 & 4 & 0.44444 & 24 & $\mathrm{~N}$
\end{tabular}

Table 1

Wave integer combinations that generate hexagonal patterns. The parameter $H$ measures the departure from regular hexagons and $N$ is the number of hexagons in the periodic box. The final column indicates whether or not the pattern has a reflection symmetry.

Consider a pattern-forming partial differential equation with a control parameter $r$ and a zero solution that exists for all $r$. Suppose that there is a marginal stability curve $r_{c}(k)$, on which the growth rate of perturbations to the zero solution vanishes, with a single minimum. There are many examples of such systems, including convection and the Swift-Hohenberg model [17]. Let $r_{0}$ be the minimum value of $r_{c}(k)$, and let $k_{0}$ be the corresponding wavenumber. Now 
for a finite box size $L$, only discrete wavenumbers given by (3) can occur, and in general these will not include $k_{0}$. For large values of $L$ however, wavenumbers close to $k_{0}$ will be included, and the separation between wavenumbers in the vicinity of $k_{0}$ scales as $1 / L^{2}$. This allows a small parameter $\epsilon$ to be introduced, representing the difference between $k_{0}$ and the three wavenumbers that generate the hexagonal pattern. With the scaling $k_{i}=k_{0}+O(\epsilon)$ for $i=1,2,3$, the corresponding values of $r_{c}$ are $r_{c i}=r_{o}+O\left(\epsilon^{2}\right)$, since $r_{0}$ is the minimum of $r_{c}(k)$. Writing $r=r_{0}+\epsilon^{2} r_{2}$, all growth rates are $O\left(\epsilon^{2}\right)$ so the appropriate time scale for the amplitude equations is $T=\epsilon^{2} t$.

It is now possible to write down the governing amplitude equations for the three Fourier modes obeying the resonance condition (4). Writing

$$
w=\sum_{1}^{3} A_{j} \exp \left(2 \pi i\left(m_{j} x+n_{j} y\right) / L\right)
$$

it can be shown that the sum of the phases of the $A_{j}$ tends to zero [12], so that with an appropriate choice of origin the amplitudes $A_{j}$ can be taken to be real. The scaled amplitude equations are then

$$
\begin{aligned}
& \dot{A}_{1}=\lambda_{1} A_{1}+A_{2} A_{3}, \\
& \dot{A}_{2}=\lambda_{2} A_{2}+A_{1} A_{3}, \\
& \dot{A}_{3}=\lambda_{3} A_{3}+A_{1} A_{2} .
\end{aligned}
$$

Here, the dot represents the rate of change on the slow timescale $T$, the linear coefficients $\lambda_{1}, \lambda_{2}$ and $\lambda_{3}$ are proportional to $\left(r-r_{c j}\right) / \epsilon^{2}$ and the amplitudes $A_{i}$ have been scaled by a factor $\epsilon^{2}$. With these scalings, all terms in (15) are of the same order, and any cubic terms in the amplitude equations will be smaller by a factor $\epsilon^{2}$.

Note that the coefficients of the quadratic terms in (15) are equal. This is because (15) represents a small perturbation from the case of regular hexagons. The coefficients of the quadratic terms can be set to 1 by scaling the amplitudes $A_{j}$ appropriately.

The dynamics of the system (15) depends crucially on whether there are any additional symmetries. It is useful to begin by briefly reviewing the well-known case of regular hexagons, for which $\lambda_{1}=\lambda_{2}=\lambda_{3}$. In this case the fixed points of (15) are the solution $A_{1}=A_{2}=A_{3}=0$, which is stable for $\lambda_{1}<0$ and unstable for $\lambda_{1}>0$, and four equivalent hexagonal solutions $A_{1}= \pm \lambda_{1}$, $A_{2}= \pm \lambda_{1}, A_{3}=-A_{1} A_{2} / \lambda_{1}$, for which the three eigenvalues are $2 \lambda_{1}, 2 \lambda_{1}$ and $-\lambda_{1}$. The bifurcation at $\lambda_{1}=0$ is transcritical, and the hexagonal solution is never stable. 
In the case where $\lambda_{1}=\lambda_{2}$, two of the wavenumbers are equal in magnitude and the resultant pattern has a reflection symmetry (e.g. Fig. 1a). The fixed points are

(i) $A_{1}=A_{2}=A_{3}=0$. This is stable if $\lambda_{1}<0$ and $\lambda_{3}<0$, and undergoes stationary bifurcations at $\lambda_{1}=0$ and $\lambda_{3}=0$.

(ii) $A_{1}= \pm \sqrt{\lambda_{1} \lambda_{3}}, A_{2}= \pm \sqrt{\lambda_{1} \lambda_{3}}, A_{3}=-A_{1} A_{2} / \lambda_{3}$. These four equivalent solutions only exist when $\lambda_{1} \lambda_{3}>0$. Its eigenvalues $s$ obey $s=2 \lambda_{1}$ or $s^{2}-$ $\lambda_{3} s-2 \lambda_{1} \lambda_{3}=0$. The product of roots of this quadratic is negative so this solution can never be stable.

For the case where all three linear terms in (15) are different, the three wavenumbers are different and the hexagonal pattern does not have mirror symmetry (e.g. Fig. 1b). The fixed points are

(i) $A_{1}=A_{2}=A_{3}=0$. This is stable if $\lambda_{1}<0, \lambda_{2}<0$ and $\lambda_{3}<0$, with stationary bifurcations at $\lambda_{1}=0, \lambda_{2}=0$ and $\lambda_{3}=0$,

(ii) $A_{1}= \pm \sqrt{\lambda_{2} \lambda_{3}}, A_{2}= \pm \sqrt{\lambda_{1} \lambda_{3}}, A_{3}=-A_{1} A_{2} / \lambda_{3}$. Again there are four solutions of this type. This solution only exists when either $\lambda_{1}<0, \lambda_{2}<0$, $\lambda_{3}<0$ or $\lambda_{1}>0, \lambda_{2}>0, \lambda_{3}>0$. The eigenvalues $s$ obey $s^{3}-\left(\lambda_{1}+\lambda_{2}+\right.$ $\left.\lambda_{3}\right) s^{2}+4 \lambda_{1} \lambda_{2} \lambda_{3}=0$. Since there is no linear term, the three eigenvalues obey $s_{1} s_{2}+s_{2} s_{3}+s_{3} s_{1}=0$ which shows that the solution can never be stable.

\subsection{Amplitude equations including cubic terms}

The analysis of the previous section, although asymptotically correct, has two drawbacks. Firstly, no stable nonlinear solutions are found, and secondly, solutions in the form of rolls (involving a single wavenumber only) are not found. These problems can be overcome by the addition of cubic terms to the amplitude equations. In general, this is inconsistent, since quadratic and cubic terms can only appear together if the amplitudes are $O(1)$, in which case terms of all order should appear in the equations. However, a consistent scaling can be obtained if the asymmetry in the problem (and hence the quadratic term) is small; this is a commonly used assumption $[1,7,10]$. The appropriate scaling is that the coefficient of the quadratic term is $O(\epsilon)$ (recall that $\epsilon$ is the scale of the difference between the wavenumbers). The growth rates are $O\left(\epsilon^{2}\right)$ as before, and a consistent balance between linear, quadratic and cubic terms is obtained if the amplitude scaling is $A_{i}=O(\epsilon)$. The scaled amplitude equations then become

$$
\begin{aligned}
& \dot{A}_{1}=\lambda_{1} A_{1}+A_{2} A_{3}-A_{1}\left(A_{1}^{2}+\beta A_{2}^{2}+\beta A_{3}^{2}\right) \\
& \dot{A}_{2}=\lambda_{2} A_{2}+A_{1} A_{3}-A_{2}\left(A_{2}^{2}+\beta A_{3}^{2}+\beta A_{1}^{2}\right) \\
& \dot{A}_{3}=\lambda_{3} A_{3}+A_{1} A_{2}-A_{3}\left(A_{3}^{2}+\beta A_{1}^{2}+\beta A_{2}^{2}\right) .
\end{aligned}
$$


It can be assumed that the quadratic and cubic terms are equal in each equation because the system (16) represents a small perturbation from the equations for regular hexagons; any deviation appears at higher order. By choosing an appropriate scaling for both time and the amplitudes, the coefficients of the quadratic terms and the $A_{i}^{3}$ terms have been set to unity. The constant $\beta$ is problem-dependent and cannot be scaled out. For simplicity it is assumed that $\beta>1$. This means that rolls are stable in the absence of the quadratic term. This is indeed the case for both Rayleigh-Benard convection and for the Swift-Hohenberg equation.

It is helpful to consider first the familiar case of regular hexagons for which $\lambda_{1}=\lambda_{2}=\lambda_{3}$ [6]. The bifurcation diagram for this case is shown in Fig. 2 . Solutions in the form of rolls (e.g. $A_{1}= \pm \sqrt{\lambda_{1}}, A_{2}=A_{3}=0$ ) are stable for $\lambda_{1}>1 /(\beta-1)^{2}$. Hexagons (with $A_{1}=A_{2}=A_{3} \neq 0$ ) appear at a transcritical bifurcation from the trivial solution where they are unstable, but gain stability though a saddle-node bifurcation at $\lambda_{1}=-1 /(4+8 \beta)=\lambda_{\mathrm{SN}}$ and are stable for $\lambda_{\mathrm{SN}}<\lambda_{1}<\lambda_{\mathrm{TR}}=(\beta+2) /(\beta-1)^{2}$. There are two regions of overlapping stable solutions and hence hysteresis; for $\lambda_{1}$ small and negative both the trivial solution and hexagons are stable, while for larger $\lambda_{1}$ both hexagons and rolls are stable. The rolls and hexagons are linked via a branch of mixed modes (e.g. $A_{1}=A_{2} \neq A_{3}$ ) which are always unstable. The upper stability boundary of hexagons at $\lambda_{1}=\lambda_{\mathrm{TR}}$ occurs at a transcritical bifurcation with $D_{3}$ symmetry, where the hexagons and mixed modes have a double zero eigenvalue; a centre manifold reduction near this point yields the normal form

$$
\dot{x}=\mu x+x^{2}-y^{2}, \quad \dot{y}=\mu y-2 x y
$$

with the symmetries of rotation through $2 \pi / 3$ and reflection $y \rightarrow-y$. This system is discussed in more detail later.

The procedure for analysing (16) is similar to that for (15). The behaviour depends on whether or not any of $\lambda_{1}, \lambda_{2}$ and $\lambda_{3}$ are equal, so these two cases are studied separately. Since the equations (16) have been considered by other authors $[12,7]$, the details of the calculations are not given. Attention is focussed on points not described by the previous work. The case of symmetric patterns with two equal wavenumbers is described in section 3.2 and the asymmetric case with three different wavenumbers is discussed in section 3.3.

\subsection{Patterns with two equal wavenumbers}

The fixed points of (16) when $\lambda_{1}=\lambda_{2}$ are as follows.

(i) $A_{1}=A_{2}=A_{3}=0$. This is stable if $\lambda_{1}<0$ and $\lambda_{3}<0$, and undergoes stationary bifurcations at $\lambda_{1}=0$ and $\lambda_{3}=0$. 


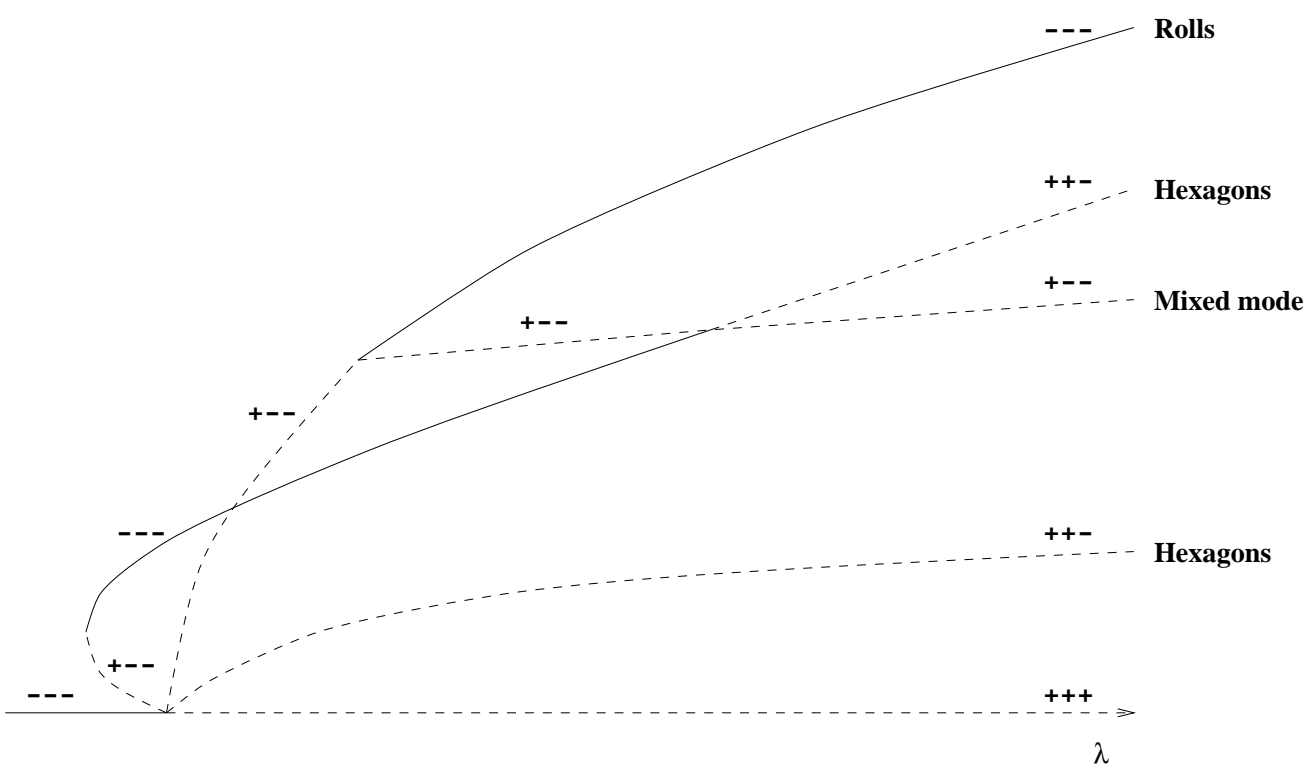

Fig. 2. Bifurcation diagram for regular hexagons. Solid lines indicate stable solutions and dashed lines represent unstable solutions. Pluses and minuses show the signs of the three eigenvalues of each branch.

(ii) ' $\lambda_{1}$-rolls' with $A_{1}= \pm \sqrt{\lambda_{1}}, A_{2}=A_{3}=0$; or $A_{2}= \pm \sqrt{\lambda_{1}}, A_{1}=A_{3}=0$. These rolls are appear at a supercritical pitchfork bifurcation and are stable if $\lambda_{1}>\left(1-\lambda_{3}+\lambda_{3} \beta\right) / \beta(\beta-1)$.

(iii) ' $\lambda_{3}$-rolls' with $A_{1}=A_{2}=0, A_{3}= \pm \sqrt{\lambda_{3}}$. These rolls also bifurcate supercritically, are stable if $\lambda_{1}<\beta \lambda_{3}-\sqrt{\lambda_{3}}$, and have bifurcations at $\lambda_{1}=$ $\beta \lambda_{3} \pm \sqrt{\lambda_{3}}$.

(iv) A mixed mode which can be regarded as hexagons, for which $A_{1}= \pm A_{2}$. This solution also branches from the zero solution at a pitchfork bifurcation at $\lambda_{1}=0$, which is supercritical if $1+\beta+1 / \lambda_{3}>0$. At onset, $A_{3}$ is much smaller than $A_{1}$ and $A_{2}$, so this pattern has a rectangular appearance. This solution can undergo saddle-node bifurcations, and branches from the $\lambda_{3}$-rolls at $\lambda_{1}=\beta \lambda_{3} \pm \sqrt{\lambda_{3}}$.

(v) A mixed mode in which all the amplitudes are different. This solution branches from the $\lambda_{1}$-rolls at $\lambda_{1}=\left(1-\lambda_{3}+\lambda_{3} \beta\right) / \beta(\beta-1)$, and can never be stable.

To draw the bifurcation diagram, the number of parameters can be reduced to one by supposing that $\beta$ and the box size $L$ are fixed but $r$ is allowed to vary. This is equivalent to writing $\lambda_{1}=\lambda_{3}+\delta$, with $\delta$ fixed. There are two possible cases, according to the sign of $\delta$, and these are shown in Fig. 3 and Fig. 4. In the bifurcation diagrams it is assumed that $\delta$ is small, so that the bifurcations from the zero solution are close together.

In the case $\delta>0$ (Fig. 3) the double bifurcation from the origin occurs first as $\lambda_{1}$ is increased. If $\delta<1 /(1+\beta)$, a mixed mode with $A_{1}= \pm A_{2}$ bifurcates subcritically from the origin and then gains stability in a saddle-node 


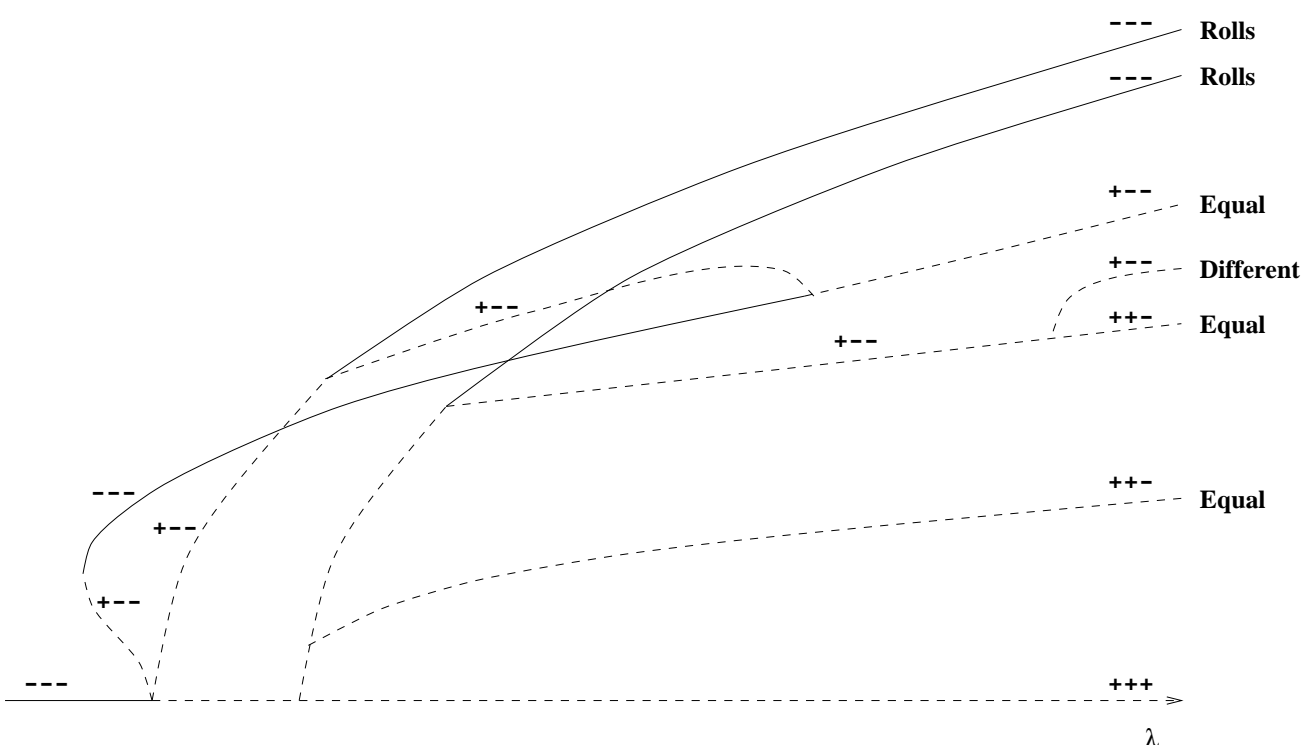

Fig. 3. Bifurcation diagram for hexagons with mirror symmetry in the case where the double mode bifurcates first $(\delta>0)$. The mixed modes are labelled according to whether all three amplitudes are different, or two are equal.

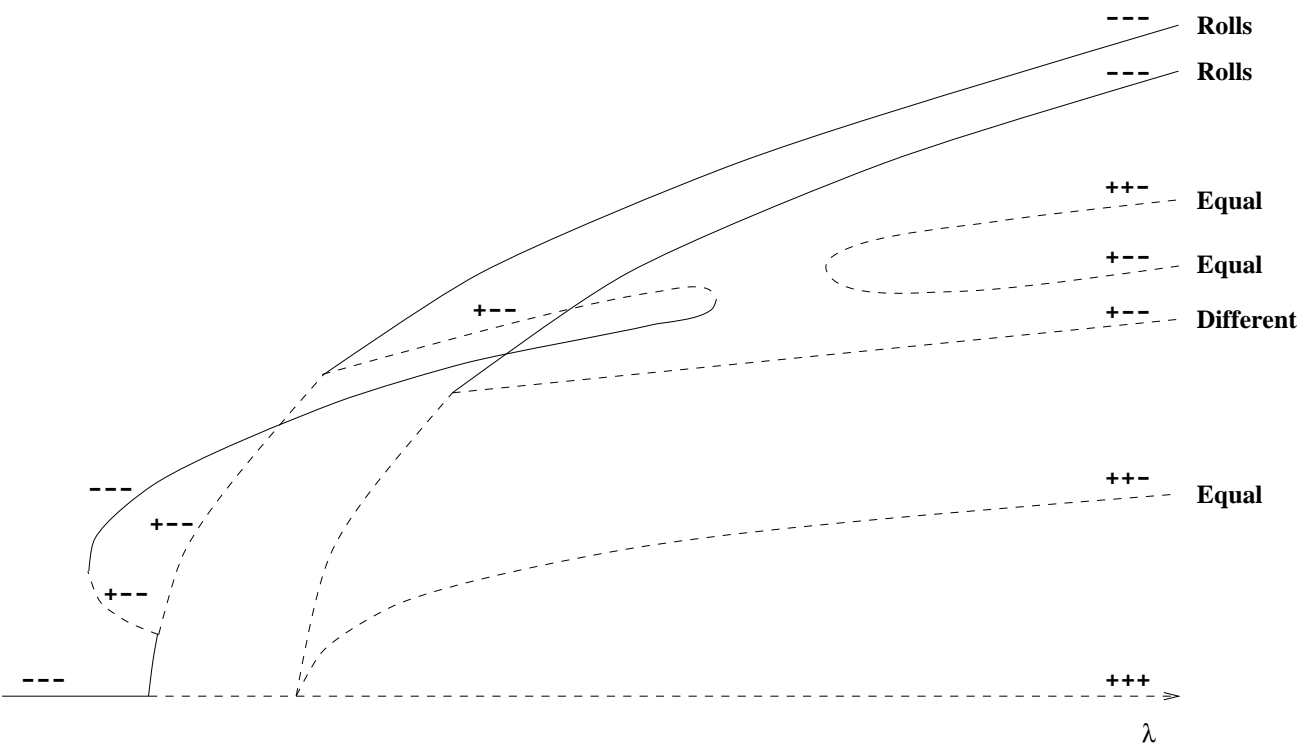

Fig. 4. Bifurcation diagram for hexagons with mirror symmetry in the case where the single mode bifurcates first $(\delta<0)$.

bifurcation. For $\delta>1 /(1+\beta)$, the mixed mode bifurcates supercritically. This mixed mode loses stability when it bifurcates to the (unstable) solution with all amplitudes different. Both roll solutions become stable at large $\lambda_{1}$.

For $\delta<0$ (Fig. 4), $\lambda_{3}$-rolls are stable at small amplitude but lose stability at a pitchfork bifurcation to the mixed mode with $A_{1}= \pm A_{2}$. This bifurcation is subcritical for small values of $|\delta|$. The mixed mode gains stability at a saddlenode bifurcation but then loses stability at a second saddle-node bifurcation 
and rejoins the roll branch, at which point the rolls regain stability. For larger values of $|\delta|$ the region of stable mixed modes decreases and for $\delta<-1 / 4(\beta-1)$ there are no stable mixed modes, so that rolls are always stable.

It is of interest to note that these diagrams can be determined to a considerable extent just by considering the splitting of the hexagonal case (Fig. 2) induced by the symmetry-breaking. The single branch of rolls splits into two branches, and both hexagon branches become mixed modes with $A_{1}= \pm A_{2}$. The mixed mode with $A_{1}= \pm A_{2}$ is unaltered, while that with $A_{1}= \pm A_{3}$ becomes a mixed mode with all amplitudes different. It follows that in both the cases $\delta>0$ and $\delta<0$, at large $\lambda_{1}$ there must be two stable roll solutions, three mixed modes with $A_{1}= \pm A_{2}$ (two with a ++- stability and one with +-- ), and one mixed mode with all amplitudes different. This approach can be used to construct Figs. 3 and 4 except near $\lambda_{1}=0$, where the amplitudes are small, and near $\lambda_{1}=\lambda_{\mathrm{TR}}=(\beta+2) /(\beta-1)^{2}$. Near this latter point, where in the case of regular hexagons there is a transcritical bifurcation (17) with $D_{3}$ symmetry, the rotational symmetry is broken, but the reflection symmetry $(y \rightarrow-y)$ is retained. A centre manifold reduction of (16) near $\lambda_{\text {TR }}$ for small $\delta$ leads to the normal form

$$
\dot{x}=\mu x+x^{2}-y^{2}-\delta, \quad \dot{y}=\mu y-2 x y,
$$

where $\mu$ is proportional to $\lambda_{1}-\lambda_{\mathrm{TR}}$ and $x$ and $y$ are rescaled forms of $2 A_{3}-$ $A_{1}-A_{2}$ and $A_{1}-A_{2}$ respectively. In the case of regular hexagons $(\delta=0)$ three mixed modes cross through the hexagons as $\mu$ passes through zero and the phase portraits are as shown in Fig. 5(a). For $\delta \neq 0$ the behaviour of (18) depends on the sign of $\delta$. For $\delta>0$, two pitchfork bifurcations occur at $\mu= \pm 2 \sqrt{\delta / 3}$ and the sequence of phase portraits is as shown in Fig. 5(b). For $\delta<0$, two saddle-node bifurcations occur at $\mu= \pm 2 \sqrt{-\delta}$; the phase portraits are shown in Fig. 5(c). This analysis enables the correct connections to be made to the various branches in the Figs. (3) and (4).

\subsection{Patterns with three different wavenumbers}

In the case where the pattern involves three different wavenumbers, $\lambda_{1}, \lambda_{2}$ and $\lambda_{3}$ are all different. The fixed points of (16) are:

(i) $A_{1}=A_{2}=A_{3}=0$. This is stable if $\lambda_{1}<0, \lambda_{2}<0$ and $\lambda_{3}<0$, and undergoes stationary bifurcations at $\lambda_{1}=0, \lambda_{2}=0$ and $\lambda_{3}=0$.

(ii) Three branches of rolls, for example $A_{1}= \pm \sqrt{\lambda_{1}}, A_{2}=A_{3}=0$. These are stable if $\lambda_{1}+\lambda_{2}<2 \beta \lambda_{1}$ and $\left(\lambda_{2}-\beta \lambda_{1}\right)\left(\lambda_{3}-\beta \lambda_{1}\right)>\lambda_{1}$.

(iii) A mixed mode in which the three amplitudes are all different, which bifurcates from the roll solutions. 
(a)
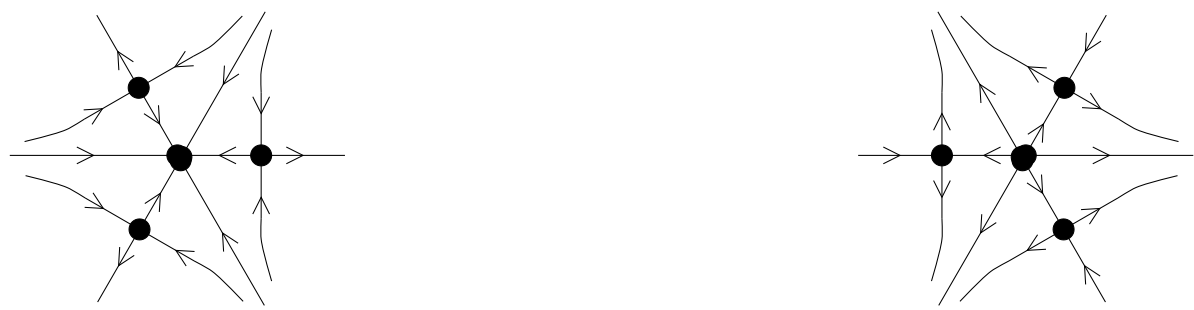

(b)
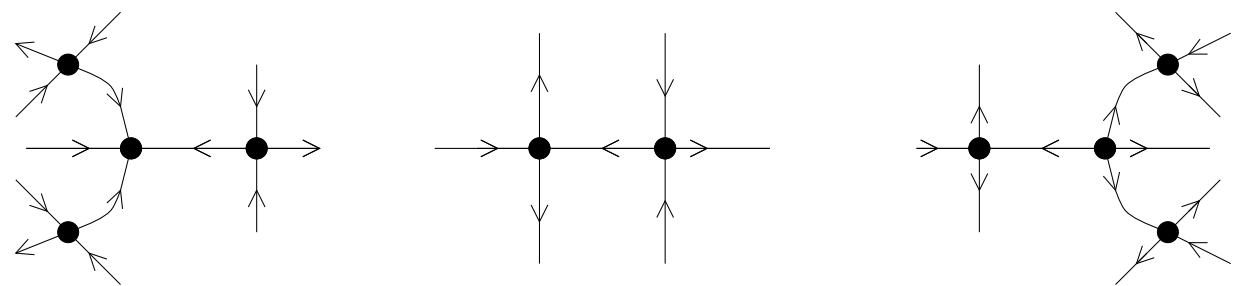

(c)
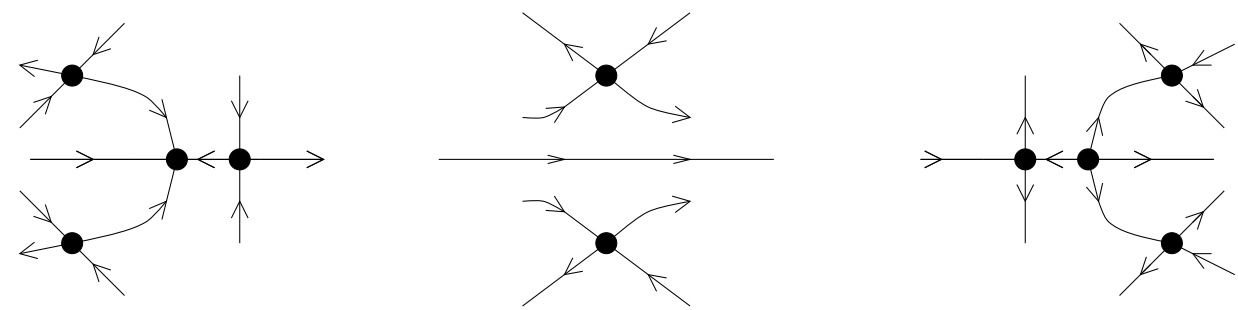

Fig. 5. The splitting or 'unfolding' of the transcritical bifurcation with broken $D_{3}$ symmetry (18). Phase diagrams are shown with $\mu$ increasing from left to right, for the three cases: (a) $\delta=0$ : Three mixed modes cross through the hexagons. (b) $\delta>0$ : Two mixed modes with all amplitudes different disappear at a pitchfork bifurcation with a mixed mode with two amplitudes equal, then reappear at a pitchfork bifurcation with the other mixed mode. (c) $\delta<0$ : The two mixed modes with two amplitudes equal disappear and then reappear at two saddle-node bifurcations.

With a fixed box size, we can set $\lambda_{1}=\lambda_{2}+\alpha=\lambda_{3}+\delta$ with $\alpha$ and $\delta$ fixed. Since the three modes are equivalent, it can be assumed without loss of generality that $\delta>\alpha>0$, so that the rolls bifurcate in the order $A_{1}, A_{2}, A_{3}$ as $\lambda_{1}$ increases. The following results can then be obtained for the roll solutions: The first branch of rolls to bifurcate is stable at onset. For small $\alpha$ and $\delta$ these rolls lose stability and then regain stability as $\lambda_{1}$ increases; for larger $\alpha$ and $\delta$ these rolls are always stable. The second branch of rolls to bifurcate is unstable at onset and becomes stable at a bifurcation to a mixed mode. The third branch of rolls is also unstable at onset, has two bifurcations to mixed modes and is then stable.

The bifurcation diagram for small $\alpha$ and $\delta$ is shown in Fig. 6. There are up to five distinct branches of mixed modes. Three of these correspond to the mixed modes in Fig. 2 and the other two correspond to the hexagons. Near 


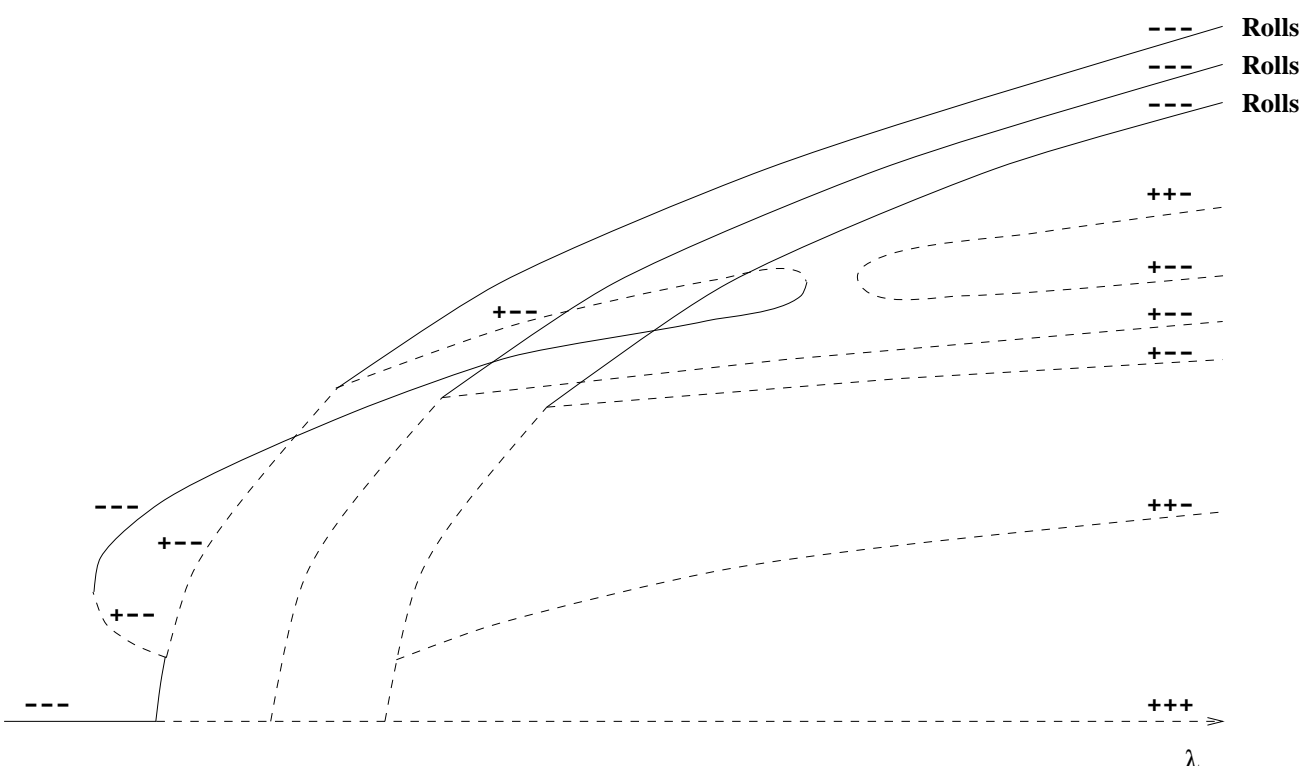

Fig. 6. Bifurcation diagram for hexagons without mirror symmetry.

the transcritical bifurcation at $\lambda_{1}=\lambda_{\mathrm{TR}}$ a centre manifold reduction yields a second-order system analogous to (18):

$$
\dot{x}=\mu x+x^{2}-y^{2}-\gamma_{x}, \quad \dot{y}=\mu y-2 x y-\gamma_{y} .
$$

Here, both the rotation and reflection symmetries have been broken. The constants $\gamma_{x}$ and $\gamma_{y}$ are related to $\alpha$ and $\delta$ but their value is not important. The behaviour of (19) is similar to that shown in Fig. 5(c) (but without the mirror symmetry), so that two of the mixed modes undergo a pair of saddlenode bifurcations, while two undergo no bifurcations, as shown in Fig. 6.

\section{Numerical simulations of the asymmetric Swift-Hohenberg equa- tion}

To illustrate the results of the preceding work, this section describes numerical simulations of the Swift-Hohenberg equation [17] modified by the addition of a quadratic term:

$$
\frac{\partial w}{\partial t}=r w-\left(1+\nabla^{2}\right)^{2} w+s w^{2}-w^{3}
$$

This equation is probably the simplest with the required properties of a preferred wavenumber $(k=1)$ and an asymmetry between $u$ and $-u$, and (20) or variations of it are commonly used as models for pattern formation (e.g. [10]). A Fourier mode with wavenumber $k$ has growth rate $r-\left(1-k^{2}\right)^{2}$. By 
(a)

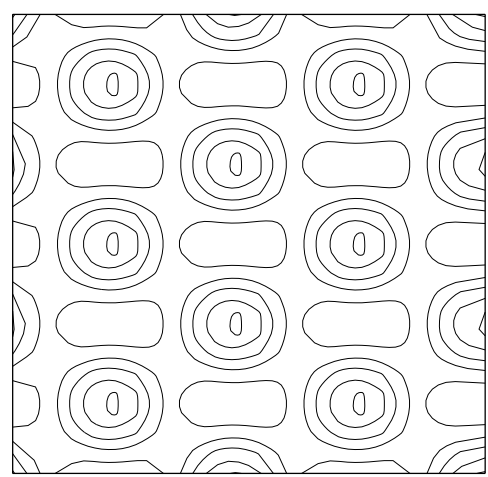

(b)

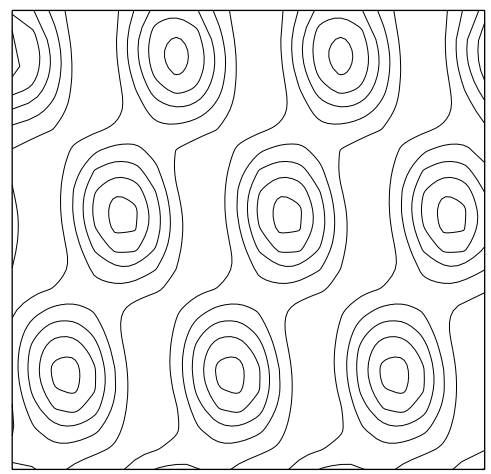

Fig. 7. Numerical solutions to (20) with $s=0.25$. (a) $r=0.03, L=23$, showing 12 irregular hexagons. (b) $r=0.05, L=21.25$, showing 9 irregular hexagons.

substituting three Fourier modes of the form (14) into (20), the amplitude equations (16) are obtained with $\beta=2$ and $\lambda_{i}=3\left(r-\left(1-k_{i}^{2}\right)^{2}\right) / 4 s^{2}$.

A Fourier spectral method was used to solve (20) with periodic boundary conditions in the region $0<x, y<L$. In Fourier space the linear parts of (20) can be solved exactly since the Fourier modes decouple. The nonlinear terms were integrated using the second-order Adams-Bashforth method. The initial condition chosen was a small-amplitude random perturbation to the zero solution.

For the case of hexagons with reflection symmetry, the pattern with 12 hexagons with wave integers $(4,0),(-2,3)$ and $(-2,-3)$ was studied. In this case, there are two different possible bifurcation diagrams (Figs. 3 and 4) according to whether the double mode or the single mode bifurcates first. For $L=23$, the $(-2,3)$ and $(-2,-3)$ modes bifurcate first and therefore the appropriate bifurcation diagram is Fig. 3. However for these parameters, $\delta=0.445$, so from the analysis of section 3.2 we expect the bifurcation to irregular hexagons to be supercritical. The numerical results show 12 hexagons for $0.002 \leq r \leq 0.03$ and pure $(3,2)$ rolls for $r \geq 0.05$, with both solutions stable for $r=0.035$ and $r=0.04$. This is consistent with Fig. 3. The solution for $r=0.03$ is shown in Fig. 7(a).

For $L=24.5$, the $(4,0)$ mode bifurcates first and the appropriate bifurcation diagram is Fig. 4. Rolls with wave integers $(4,0)$ were found for $0<r \leq 0.01$ and $r \geq 0.025$, and a hexagonal pattern including the $(-2,3)$ and $(-2,-3)$ modes was found for $0.015 \leq r \leq 0.02$. 


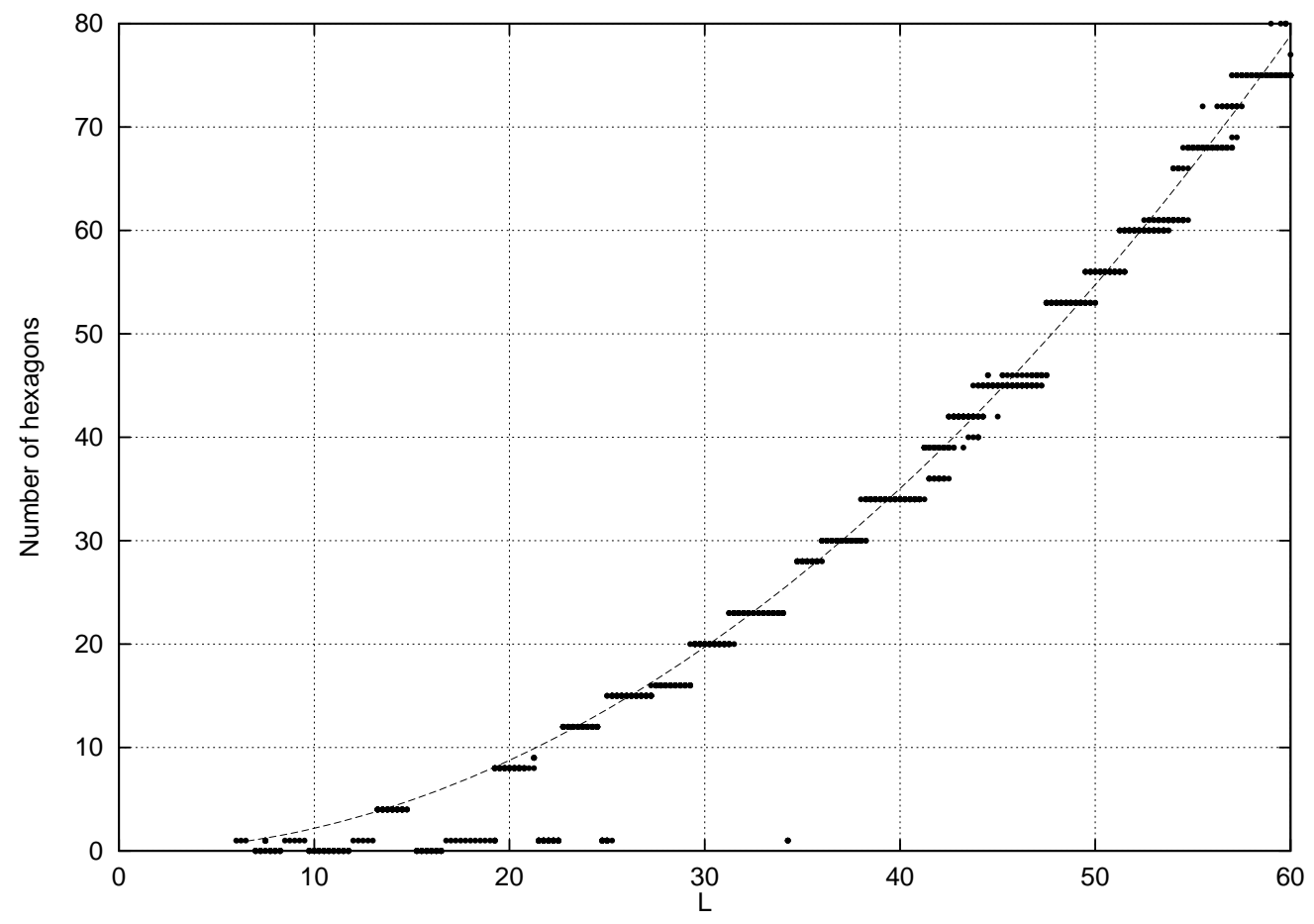

Fig. 8. Number of hexagons $N$ against box size $L$ for the equation (20) with $r=0.02$ and $s=0.25 . N=0$ indicates that the zero solution is stable and $N=1$ indicates that rolls are stable. The dashed line is the curve $N=\sqrt{3} L^{2} / 8 \pi^{2}$, obtained by dividing the area of the box by the area of a regular hexagon.

The solution with 9 hexagons, involving the wave integers $(3,2),(-3,1)$ and $(0,-3)$ was chosen to study the case without reflection symmetry. This solution was found when the box size $L$ was 21.25, and at this value of $L$ the $(-3,1)$ mode bifurcates first. A solution with 9 hexagons was found for $0.02 \leq r \leq 0.055$ and rolls with wave integers $(3,1)$ were found for $r \geq 0.06$. For $r=0.057$ both solutions were found to be stable. Referring to the bifurcation diagram (Fig. 4), rolls are also expected for small $r$; in fact however a hexagonal solution with 8 hexagons was found for $r \leq 0.02$. Interactions between hexagonal patterns with different numbers of hexagons are of course beyond the scope of the analysis of section 3. The solution for $r=0.05$ is shown in Fig. 7(b).

One question of interest which is difficult to approach analytically is which of the many possible hexagonal solutions listed in table 1 are observed as the box size $L$ is increased. To investigate this question a sequence of runs was carried out with $r=0.02$ and $s=0.25$. For each value of $L$, the computation was continued until a stationary solution was obtained. By looking at the dominant Fourier modes the solution was then identified as either rolls or hexagons and the number of hexagons was determined using (11). Since there is the possibility of multiple stable states, the computation was repeated ten times for each value of $L$, with a different small-amplitude random initial 
condition in each case. Values of $L$ between 6 and 60 were used, with a step of 0.25 in $L$. The results are summarised in Fig. 8, which plots the number of hexagons against the box size. Where the number of hexagons is zero this indicates the zero solution, and a solution in the form of rolls is shown as one hexagon. Note that there are regions in which both rolls and hexagons are stable, and also regions in which two or even three different types of hexagons are stable.

Of the hexagonal solutions in table 1 , those with $4,8,9,12,15,16,20,23$ hexagons were found. Note that, as might be expected, these are the solutions with low values of $H$, i.e. those that are closest to regular hexagons. There appears to be a preference for the symmetric hexagons. However this is no longer true at large values of the box size, where there are roughly as many asymmetric patterns as symmetric ones.

\section{Conclusions}

The main results of this paper are as follows. For numerical experiments with periodic boundary conditions, or laboratory experiments with Neumann boundary conditions, a perfectly regular hexagonal pattern is not permitted in a square box or any box with rational aspect ratio. Instead, patterns of irregular or 'non-equilateral' hexagons are observed. These patterns are composed primarily of three wavenumbers, and occur in two types. If two of the wavenumbers are equal then the resulting pattern has a mirror symmetry, but if all the wavenumbers are different it does not. There is a simple relationship (11) between the number of hexagonal cells in the pattern and the three wavenumbers.

The nonlinear dynamics of these patterns is controlled by the amplitude equations (16). The results of the analysis of these equations are summarised in the bifurcation diagrams of Figs. 3, 4 and 6. These provide a more complete picture of the dynamics than has been given by previous studies of the equations $[1,7,8,12]$. A useful technique for clarifying the bifurcations that occur is the centre manifold reduction near the transcritical bifurcation with $D_{3}$ symmetry. This reduces the algebra of the analysis considerably, enabling firm conclusions to be drawn regarding the connections between different solution branches.

The analysis is closely related to that of Proctor and Matthews [15] who investigated the interaction of modes with wave integers $(0,1),(1,0)$ and $(-1,-1)$, which give a pattern with the topology of hexagons (isolated upflows and connected downflows) but with square symmetry. Some of the results are similar, for example the fact that the quadratic resonances can lead to subcritical be- 
haviour and a preference for three-dimensional patterns over two-dimensional rolls.

This work was motivated by numerical simulations of compressible magnetoconvection [18], in which hexagonal patterns with six rising plumes were found. The results obtained suggest that for very small Rayleigh numbers, rolls should be stable, and that for larger Rayleigh numbers rolls should again become stable. However it must be borne in mind that the equations (16) are only valid if the up-down asymmetry (the departure from the Boussinesq approximation in the case of convection experiments) is small, and this is generally not the case. There is much more complicated dynamics in these numerical experiments and further analytical work is required to understand and interpret them fully.

\section{Acknowledgements}

I am grateful to the University of Nottingham, the Royal Society and the Nuffield Foundation for financial support. This work has benefited from discussions with S. Cox, M. Proctor, A. Rucklidge and N. Weiss.

\section{References}

[1] S.M. Cox, IMA J. Appl. Mathematics (1997).

[2] J.D. Crawford, Phys. Rev. Lett 67(4) 441-444.

[3] J.D. Crawford and E. Knobloch, Ann. Rev. Fluid Mech 23 (1991) 341-387.

[4] J.D. Crawford, J.P. Gollub and D. Lane, Nonlinearity 6 (1993) 119-164.

[5] B. Dionne, M.Silber and A.C. Skeldon, Nonlinearity, in press.

[6] M. Golubitsky, J.W. Swift and E. Knobloch, Physica D 10 (1984) 249-276.

[7] P. Hall and R.E. Kelly, Physical Review E 52 (1995) 3687-3696.

[8] R.B. Hoyle, G.B. McFadden and S.H. Davis, Phil. Trans. A 354 (1996) 29152949.

[9] E.L. Koschmieder and S.A. Prahl, J. Fluid Mech. 215 (1990) 571-583.

[10] C. Kubstrup, H. Herrero and C. Perez-Garcia, Phys. Rev. E 54 (1996) 15601569.

[11] A. Kudrolli and J.P. Gollub, Physica D 97 (1996) 133-154. 
[12] B.A. Malomed, A.A. Nepomnyashchy and A.E. Nuz, Physica D 70 (1994) 357369.

[13] P.C. Matthews, M.R.E. Proctor and N.O. Weiss, J. Fluid Mech. 305 (1995) 281-305.

[14] Pattern Formation and Instabilities in Continuous Dissipative Systems, Physica D 97 (1996).

[15] M.R.E. Proctor and P.C. Matthews, Physica D 97 (1996) 229-241.

[16] A.C. Skeldon, K.A. Cliffe and D.S. Riley, J. Comp. Phys, in press.

[17] J. Swift and P. Hohenberg, Phys. Rev. A15, (1977) 319-328.

[18] N.O. Weiss, D.P. Brownjohn, P.C. Matthews and M.R.E. Proctor, Monthly Notices of the Royal Astronomical Society 283 (1996) 1153-1164. 